\title{
The reggie/flotillin connection to growth
}

\author{
Claudia A.O. Stuermer
}

University of Konstanz, Department of Biology, 78457 Konstanz, Germany

\begin{abstract}
The proteins reggie-1 and reggie-2 were originally discovered in neurons during axon regeneration. Subsequently, they were independently identified as markers of lipid rafts in flotation assays and were hence named flotillins. Since then, reggie/flotillin proteins have been found to be evolutionarily conserved and are present in all vertebrate cells - yet their function has remained elusive and controversial. Recent results now show that reggie/flotillin proteins are indeed necessary for axon regeneration and growth: no axons form when reggies/flotillins are downregulated and signaling pathways controlling actin dynamics are perturbed. Their widespread expression and conservation, however, suggest that these proteins regulate basic cellular functions beyond regeneration. It is argued here that the reggie/flotillin proteins regulate processes vital to all cells - the targeted delivery of bulk membrane and specific membrane proteins from internal vesicle pools to strategically important sites including cell contact sites, the $T$ cell cap, regenerating axons and growth cones and other protrusions.
\end{abstract}

\section{Introduction}

Reggie-1 and reggie-2 (flotillin-2 and flotillin-1; Box 1) were discovered as neuronal proteins upregulated in retinal ganglion cells during the regeneration of axons after lesion of the optic nerve, implying that they contribute to regeneration $[1,2]$. Following their discovery it was not immediately evident how these proteins might promote axon growth and regeneration because they reside at the cytoplasmic face of the plasma membrane (Figure 1), unlike cell-surface adhesion molecules (cadherins, integrins and others) and receptors for axon guidance cues (such as netrin and slit receptors). Moreover, reggie/flotillin proteins have been found to be highly conserved from fly to human and are present in every vertebrate cell type examined so far [3,4]. Reggie/flotillin-like proteins exist in bacteria [5] and plants [6], suggesting that these proteins serve functions beyond the nervous system. In the absence of a consistent view on reggie/flotillin functions, their identification as markers of lipid rafts (Box 1) also did not explain the role they might play in axon growth and regeneration. Furthermore, they have been implicated in cell division [7], melanoma progression [8], Alzheimer and Parkinson disease (indirectly by their high expression levels in diseased brains), Wingless (wg) and Hedgehog

Corresponding author: Stuermer, C.A.O. (Claudia.Stuermer@uni-konstanz.de). (hh) signaling in Drosophila development [9] and in other phenomena (e.g. Ref. [10]).

Conflicting views on their localization and function have complicated the situation. For example, they were believed to be constituents of caveolae [11], although neurons and lymphocytes do not possess caveolae (Ref. [2] and citations therein; also Ref. [12]) but do express reggie/flotillin. Here and in other cells, reggie/flotillin proteins form their own plasma membrane microdomains (Box 1) through oligomerization by means of their C-terminal tail domain [13]. In addition, a role for reggie/flotillin proteins was implied in the endocytosis of GPI-anchored proteins through a pathway independent of clathrin-coated pits and caveolae, but instead involving reggie/flotillin-dependent invaginations and reggie/flotillin phosphorylation [14-16]. However, other investigators have demonstrated that the GPI-anchored prion protein (PrP) and the amyloid precursor protein (APP) are taken up through clathrin-coated pits, although PrP and APP were clustered in reggie/flotillin microdomains prior to endocytosis and colocalized at lysosomes [17-19]. Moreover, no reggie/flotillin-dependent invaginations were detected by other authors, phosphorylation was not observed in N2a and T cells, nor were reggie/ flotillin proteins found in early endosomes [17,20,21], and this makes it difficult to understand how reggie/flotillin mediates endocytosis of membrane proteins. However, they are observed in many cellular compartments/vesicles: the recycling compartment, multivesicular bodies and late endosomes/lysosomes, aggresomes and, importantly, at many vesicles travelling to and from the plasma membrane [17], suggesting a role for the reggie/flotillin proteins in the communication between plasma membrane and intracellular trafficking.

\section{New viewpoint}

A novel viewpoint of reggie/flotillin functions will be presented here that might allow new interpretations of earlier results and that can account for the existence of the proteins in many different cell types and organisms, their localization at intracellular vesicles and the plasma membrane and, most importantly, that can explain their role in axon growth and regeneration and account for the spectrum of functions discussed below. I propose that reggie/ flotillin microdomains promote the assembly of specific cell-surface proteins and signaling complexes at the plasma membrane in a cell-type-specific manner, thus creating a signaling center to trigger the recruitment of bulk membrane material and proteins from internal vesicular stores to specific plasma membrane domains. This 


\section{Box 1. Characteristics of the reggie/flotillin proteins}

Reggie/flotillin proteins form plasma membrane domains, the socalled reggie microdomains, and reside at the cytoplasmic face of the plasma membrane anchored through $\mathrm{N}$-terminal myristoyl and palmitoyl groups $[10,51]$ and a hydrophobic stretch in their head domain [52] (Figure 1). They promote the local co-assembly of activated and specific GPI-anchored proteins on the cell surface and allow interaction with signal transduction molecules, including the Src-family tyrosine kinases Src, Fyn, Lck and the CAP-associated signaling complex in crosstalk with the small GTPase TC10 [22]. Furthermore, reggie/flotillin proteins communicate with and bind to actin [28,34], features that reggies share with lipid rafts $[36,40]$. Reggies were independently identified as lipid raft proteins [11] that typically become enriched in the 'floating lipid raft' fraction after solubilization of membranes in cold Triton X-100 and sucrose density centrifugation - and they were hence called 'flotillins' (reggie-1 is flotillin-2, and reggie-2 is flotillin-1). Since then, flotillins have been used as lipid raft markers [11] in many biochemical studies (too numerous to cite). According to our view, however, reggie/flotillin proteins represent scaffolds of microdomains - similar to caveolin in caveolae - while being distinct from caveolae $[2,32,40]$. Reggie/ flotillin microdomains are microscopically visible and are better defined than rafts - these latter are characterized by their special lipid composition and not by scaffolding proteins, and are hence difficult to see. Reggie/flotillin proteins are distantly related to other microdomain scaffolding proteins, notably stomatin, prohibitin, podocin and erlin [53].

The reggie/flotillin microdomains of $\leqq 100 \mathrm{~nm}[32,33]$ comprise hetero-oligomers (Figure 1) associated through their C-terminal tail domains $[10,13]$, each with a predicted $\alpha$-helical coiled-coil structure $[1,3]$. The microdomains appear as puncta along the plasma membrane (Figure 3) unless they cluster into macrodomains [34,35] through a reduction of the distance between individual microdomains. Examples are the preformed reggie cap in T cells (Figure 3), the condensation of reggies along cell-cell contact sites [46] and their selective accumulation in growth cones (Figure 3 ) and growing processes in general. In addition to the plasma membrane, reggie/flotillin proteins are found in association with vesicles in intracellular compartments, namely dense core vesicles in PC12 cells, Golgi-associated vesicles, the Rab11 recycling compartment, multivesicular bodies [17], late endosomes/ lysosomes [32,35], phagosomes of macrophages [31], lipid bodies [54] and in the non-vesicular aggresomes [17] but not in early endosomes. Interestingly, reggie/flotillin vesicles have been observed to traffic rapidly bidirectionally between intracellular sites and the plasma membrane [17], a process that is accelerated by epidermal growth factor (EGF) that is known to promote lamellipodia formation, cell migration and elongation of processes. Thus, it seems that reggie/ flotillin microdomains are signaling centers engaged in crosstalk between the inner vesicle pool and the plasma membrane. new viewpoint was derived from reports on the function of reggie/flotillin proteins in fat cells known as adipocytes, $\mathrm{T}$ cells, embryonic stem cells, neurons and various cell lines, as discussed below.

\section{Reggie/flotillin and GluT4 recruitment in adipocytes}

In their effort to understand insulin signaling and the causes of diabetes, Alan Saltiel and colleagues discovered that flotillin-1/reggie-2 binds the c-Cbl-associated adaptor protein (CAP) and communicates with a CAPassociated signaling complex that activates the small guanosine 5'-triphosphate-hydrolyzing enzyme (GTPase) TC10 (Figure 2). In adipocytes, TC10 triggers the transfer of the glucose transporter GluT4 from a specific vesicular storage compartment to the plasma membrane [22,23], a process involving the exocyst and the (Ras-related) GTPase RalA [22,24]. TC10 activation occurs in lipid rafts (or reggie/flotillin microdomains; Box 1). Much like reggie/ flotillin proteins, TC10 and RalA can associate with vesicles, such as the Rab11-positive recycling compartment and (transport) vesicles en route to the plasma membrane $[22,25,26]$. This is consistent with the known

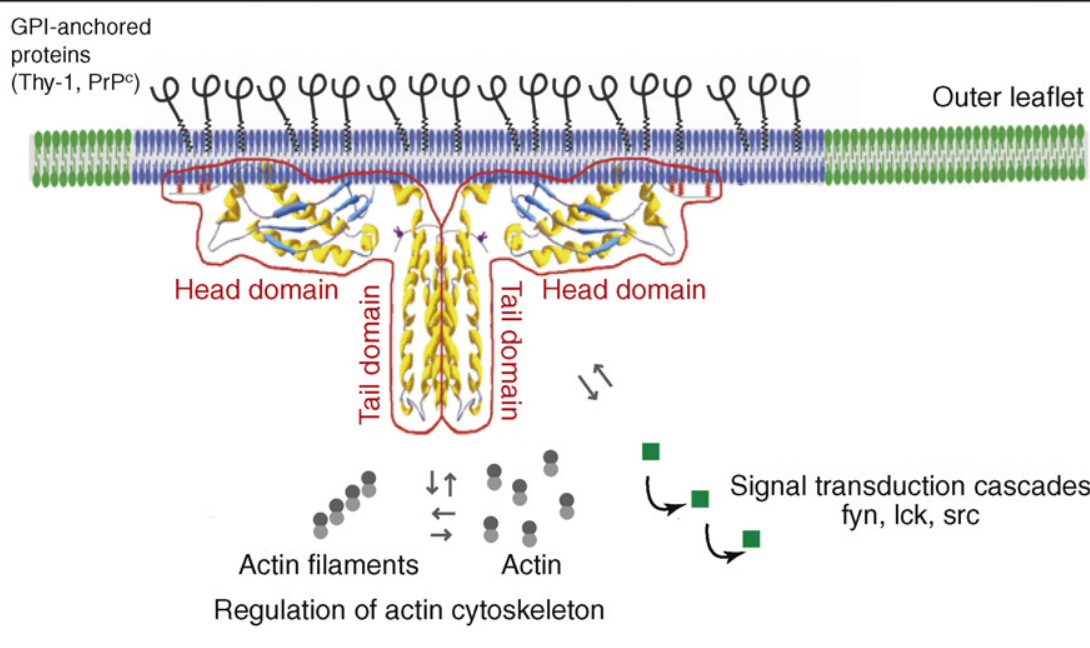

TRENDS in Cell Biology

Figure 1. Reggie/flotillin microdomains and co-clustering of the activated GPI-anchored proteins Thy-1 and PrP. Reggie-1/flotillin-2 and reggie-2/flotillin-1 (presented oversized), are illustrated as two hammer-shaped structures, back-to-back, encircled by a red line, that form homo- and hetero-oligomers at the cytoplasmic face of the plasma membrane and afford scaffolds for microdomains with lipid raft properties (depicted in blue). Acyl residues and hydrophobic amino acids in the $\mathrm{N}$-terminal head domain (NMR structure; Ref. [52]) promote reggie binding to the cytoplasmic face of the plasma membrane. The predicted alpha-helical coiled-coil domains at the Cterminus (tail domain) are involved in oligomerization. Reggies interact with Src tyrosine kinases (Src, Fyn, Lck) as well as with Rho-family GTPases through the CAPassociated signaling complex (Figure 2) and affect the dynamics of the actin cytoskeleton. Activated GPI-anchored proteins (pigtailed structures) at the outer leaflet of the plasma membrane are densely packed in the reggie microdomains. The GPI-anchored proteins communicate with signaling molecules most likely through co-clustering with reggie/flotillin (image adapted with permission from Ref. [3]; @ Cellular and Molecular Life Sciences). 


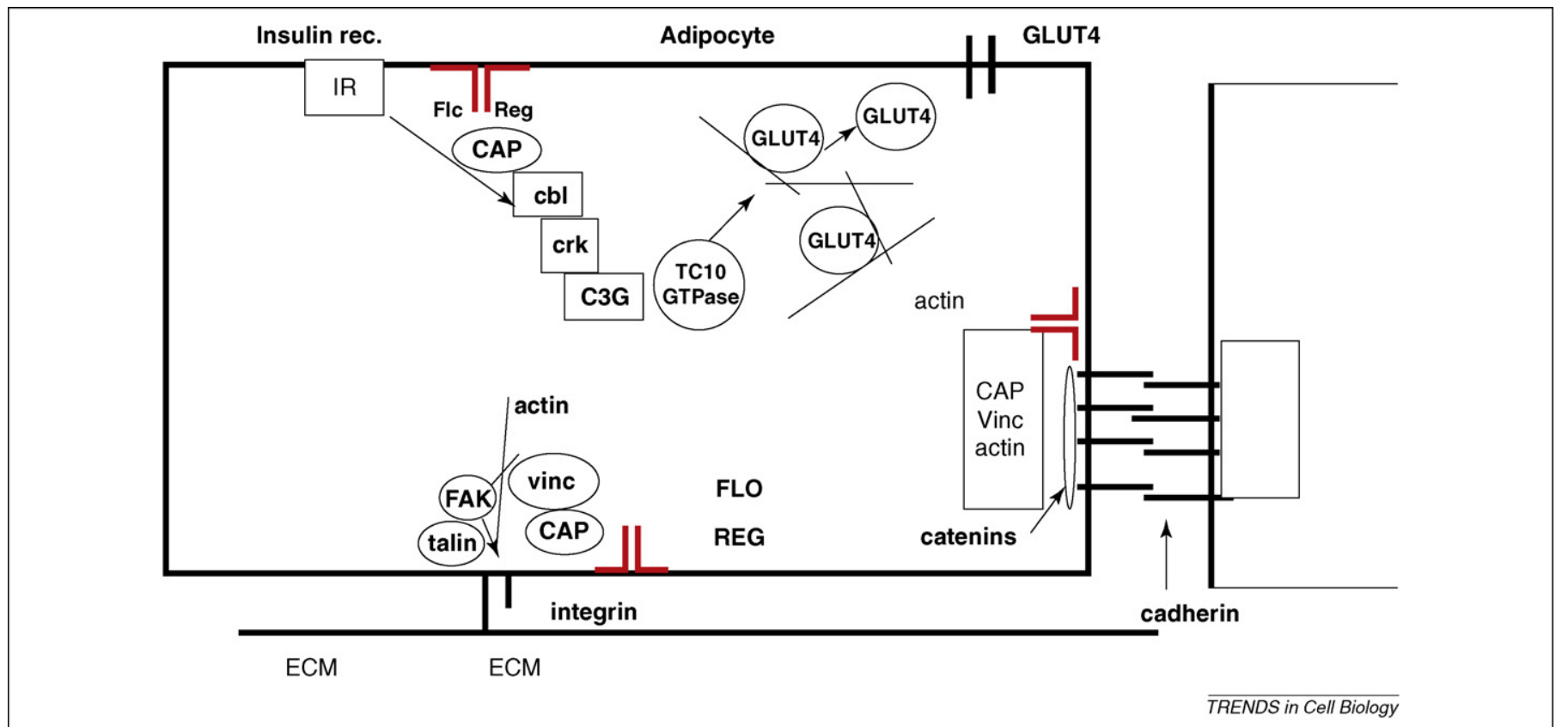

Figure 2. Involvement of reggie/flotillin (reg/flo) in the recruitment of membrane proteins such as the glucose transporter Glut4 in adipocytes, cadherins at cell-cell contact sites, and integrins at the cell-substrate interface of a model cell (after Ref. [29]). In adipocytes the protein complex associated with the reggie/flotillin-binding adaptor protein CAP activates the Rho-GTPase TC10, that then allows the vesicles with Glut4 (associated with actin, indicated by black lines) to target the plasma membrane, whereupon vesicle fusion and Glut4 integration into the plasma membrane can take place. This cascade is initiated by insulin binding to the insulin receptor (IR), that then activates $\mathrm{Cbl}$. A protein complex associated with reggie/flotillin and the adaptor protein CAP, or its relatives vinexin and ArgBP2, is suggested to interact with the cadherincatenin adhesion complex at cell-contact sites. A complex, involving reggie/flotillin, associated adaptors and FAK, colocalizes with integrins, vinculin (vinc) and talin at contacts between the cell and extracellular matrix (ECM) (image adapted from Ref. [30] and reproduced with permission).

properties of reggie proteins in activating TC10 and other small GTPases, that in turn regulate actin dynamics and vesicle trafficking [27,28]. Reggie/flotillin-decorated vesicles were observed to shuttle between the plasma membrane and more distant intracellular sites [17]. This indicates that reggie/flotillin microdomains at vesicle membranes could contribute to the signaling important for vesicle mobilization, trafficking and targeting that requires the activity of small GTPases that, in turn, have a preference for rafts [24,29].

Kioka and colleagues [30] suggest that the reggie/flotillin-binding protein $\mathrm{CAP} /$ ponsin and its relatives ArgBP2 and vinexin communicate with the actin cytoskeleton (as does reggie/flotillin; Ref. [27]) and affect not only GluT4 but also substrate- and cell-adhesion proteins, including integrins and the cadherins, implying that reggie/flotillin functions in a pathway for polarized delivery of cargo. Such cargo includes, for example, transporters (such as Glut4), cell-adhesion molecules (such as cadherins, integrins), receptors, ion channels and many other proteins that need to be targeted to specific sites. Reggie/flotillin microdomain clustering and co-clustering with membrane proteins thus provides external signals and specificity (Figure 3).

Because reggie/flotillin proteins have also been identified on intracellular vesicles [17,22,23,31], they might also form signaling centers for the clustering of signaling molecules at the vesicle membrane [31] so as to promote vesicle transport and targeted delivery. As an example, reggie/ flotillin proteins affect the distribution of Wingless (wg) and Hedgehog (hh) [9] morphogens delivered through the secretory vesicular pathway.

\section{T cell receptor recruitment}

The proposed reggie/flotillin function in the polarized delivery of cargo is supported by their distribution and function in $\mathrm{T}$ cells. In $\mathrm{T}$ cells, reggie/flotillin microdomains are preclustered at one pole of the cell, representing 'the preformed reggie cap' (Figure 3A), where the T cell receptor complex and the signaling molecules associated with $\mathrm{T}$ cell activation coalesce upon stimulation [32,33,34]. The preformed reggie cap is important for $\mathrm{T}$ cell activation, as shown by means of a dominant-negative reggie construct (R1EA) that disrupted the cap and interfered with the formation of the immunological synapse [35]. In $\mathrm{T}$ cells, one stimulus for $\mathrm{T}$ cell receptor complex assembly in the cap is the activation of GPI-anchored proteins by antibody crosslinking [36]. This applies, for instance, to Thy-1 and the cellular prion protein, PrP, whose activation by crosslinking leads to their selective association with the reggie cap [33,35]. Activated PrP in the reggie cap has been shown to elicit the phosphorylation of the MAP (mitogen-activated protein) kinase ERK1/2 and to evoke a distinct $\mathrm{Ca}^{2+}$ signal leading to the recruitment of CD3 [33,37], the major T cell receptor component, to the membrane in the cap. Although PrP clustering and the $\mathrm{Ca}^{2+}$ signal can recruit CD3, this is not sufficient for the full activation of the $\mathrm{T}$ cell receptor complex [33,35], that would require crosslinking of CD3 or stimulation by antigen. $\mathrm{T}$ cell receptor recruitment involves the communication with actin dynamics through Src tyrosine kinases (Fyn, Lck, Src), Rho GTPases and the signaling molecule Vav [38]. Thus, the microdomains consisting of reggie/flotillin oligomers (Box 1) serve as platforms for PrP cluster formation, signaling, actin rearrangement and the recruitment of CD3 (from internal stores) to the cap. 


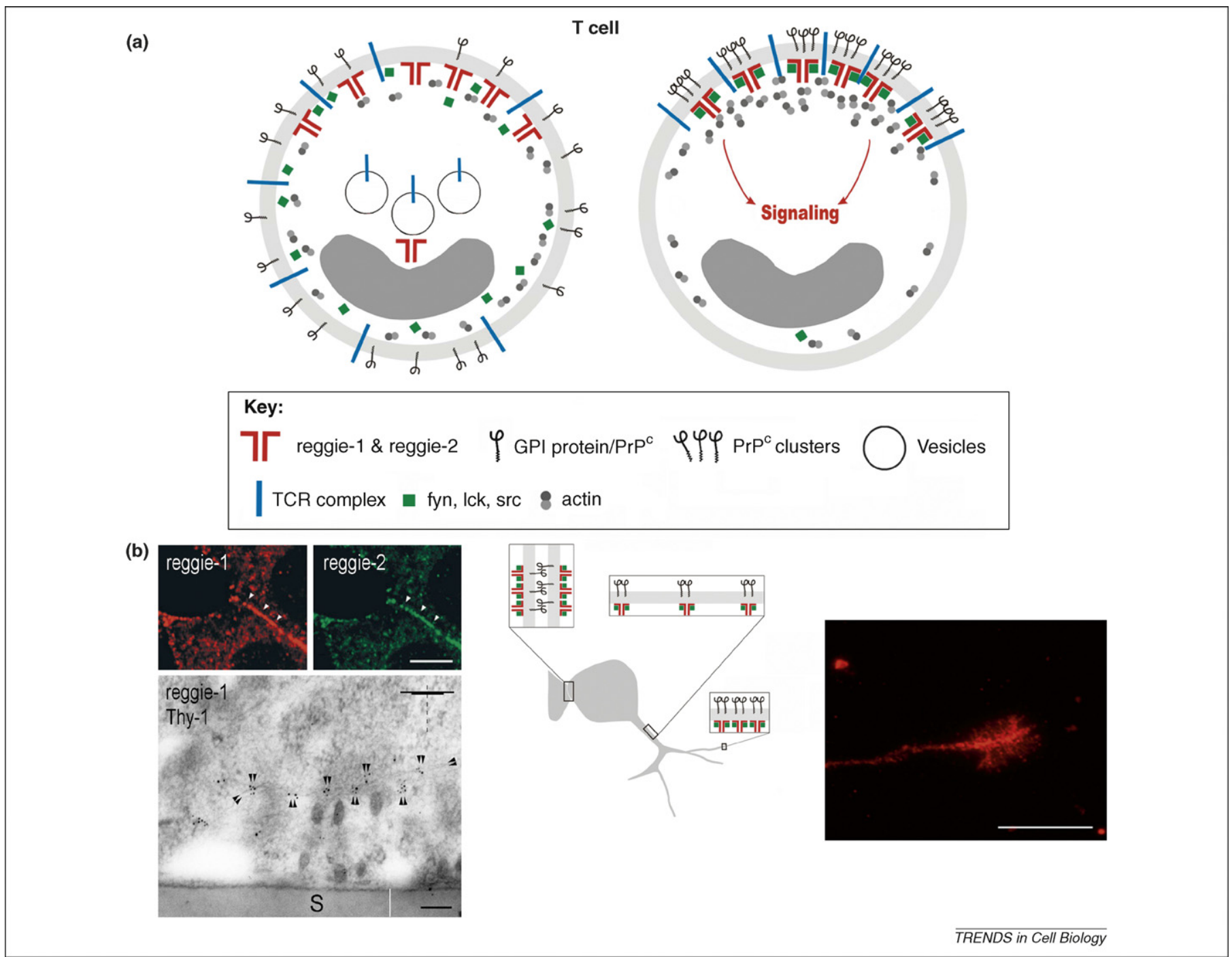

Figure 3. Reggie/flotillin microdomains cluster at cell contacts, growth cones and in the T cell cap. (a) In T cells, reggie/flotillin proteins are already clustered at one pole of the cell before T cell stimulation (to the left). Activation of the prion protein PrP by antibody crosslinking results in co-capping of PrP and reggie/flotillin (to the right) and promotes the recruitment of CD3 and the T-cell receptor complex to the cap (modified from Ref. [33] and reproduced with permission; ( $)$ The FASEB Journal). (b) The schematic neuron (in the middle of the three images) co-clusters the GPI-anchored proteins PrP or Thy-1, reggie/flotillin and Src tyrosine kinases at contact sites between cells and in growing processes, whereas reggie/flotillin microdomains are more widely spaced along the axon. The micrograph to the left represents cell contact sites after double immunostaining (reggie-1 and reggie-2) and shows dense clusters of reggie-1 and reggie-2 microdomains, whereas other domains of the cell have microdomains that are more widely spaced. Bar, $5 \mu \mathrm{m}$. Double-immunogold labelling showing co-clusters of large and small gold grains for reggie-1 and Thy-1 at cell-cell contact sites (double-headed arrowhead). S, support; the upper right-hand corner indicates the plane of section. Bar, $0.1 \mu \mathrm{m}$. Reggie-1/flotillin-2 staining is concentrated in growth cones (micrograph to the right), as opposed to the rest of the axon, and reaches into lamellipodia and filopodia (modified with permission from Ref. [46]; $\odot$ The Biochemical Society, http://symposia.biochemistry.org/). Bar, $10 \mu \mathrm{m}$.

\section{Cadherin recruitment}

Reggie/flotillin proteins are widely clustered at contact sites between cells [33,39] and so is PrP. PrP-PrP transinteractions in the contacts between cells, epithelial, neuronal and embryonic stem cells lead to PrP clustering and co-clustering with reggie/flotillin proteins. PrP clusters are residents of reggie/flotillin microdomains and seem to be crucial for cell contact formation and downstream actin re-arrangement in zebrafish embryos and other cells [26]. We have demonstrated that PrP clustering results in signaling that is necessary for the PrP-dependent recruitment of epithelial (E)-cadherin from the internal vesicle pool to the contacts between cells [39], similar to the CD3 recruitment into reggie caps [33].

Exactly how PrP communicates with reggie/flotillin for the recruitment of cadherins remains to be solved. There is ongoing discussion regarding whether proteins with lipid anchors confined to one leaflet of the lipid bilayer are capable of signal transduction without associated transmembrane proteins. Recent results from a biophysical simulation approach, however, suggest that cluster formation of GPI-anchored proteins with lipid anchors in the outer leaflet of the plasma membrane is crucial and efficient in influencing the adjacent cytoplasmic leaflet, in particular if the latter contains another cluster of proteins (such as the reggie/flotillin microdomain) with their own lipid anchors - the myristoyl- and palmitoyl-residues in reggie/flotillin (Matthias Weiss, personal communication). Moreover, lipid rafts possess a specific lipid composition containing, for instance, sphingolipids with lipid chains longer than the width of one leaflet [40]. It is thus conceivable that co-clusters of proteins in the outer and inner 
leaflet can transduce signals into the cell, activating Srctyrosine kinases that are also lipid-anchored and interact with reggie/flotillin, such as Fyn [41]. Nevertheless, in biological membranes, the participation of transmembrane proteins in signal transduction cannot be excluded.

\section{Summary of cell-surface protein reggie interactions}

Together, insights from adipocytes, $\mathrm{T}$ cells, zebrafish embryonic stem cells and cell lines shed new light on the function of $\operatorname{PrP}$ [42] as well as that of reggie/flotillin. They support the model that reggies are platforms necessary for the assembly of specific cell-surface proteins, for signal transduction and the activation of small GTPases so as to regulate actin dynamics. The specificity and the trigger for signaling appear to originate from the cell-surface associates of reggie/flotillin microdomains that are cell-type dependent (insulin receptor in adipocytes, $\mathrm{PrP}$ in T cells and at cell contact sites) and domain specific (the cap in $\mathrm{T}$ cells, contact sites). The cell-surface-derived signals often (or always) target GTPases and actin dynamics, conceivably to activate and recruit specific vesicles from the intracellular pool. Reggie/flotillin proteins would thus be relevant for many different cell types and at the same time for celltype-specific actions - contact formation, adhesion, cell differentiation and division, cell process formation and polarization, as well as axon growth and regeneration. Such a view would account for the fact that reggie/flotillin proteins are ubiquitous and, at the same time, can be involved in specific functions in widely different cell types. How do reggie/flotillin proteins perform such tasks in neurons?

\section{Reggies regulate axon regeneration}

Upon lesioning the optic nerves of fish, the retinal ganglion cells (RGCs) upregulate growth-associated proteins and regenerate their axons to enable the recovery of vision (Box 2) - a remarkable capability - unfortunately one that is not conserved in warm-blooded vertebrates [43]. More than a dozen growth-associated proteins in RGCs have been identified at the molecular level, and these were recently complemented by a proteomics approach in mammalian cortical neurons [44]. In this context, the reggie proteins

\section{Box 2. Axon regeneration in the central nervous system (CNS)}

Unlike mammals, teleost fishes (e.g. zebrafish, goldfish) are able to regenerate CNS axons after fiber tracts in the spinal cord or optic nerve are lesioned [42]. Although the molecular events underlying success and failure of axon regeneration are insufficiently understood, some key features have nevertheless been identified. One explanation for efficient regeneration in teleosts, but not in mammals, is the glial cell environment of the lesioned axons - this has been found to be inhibitory for growth cones in the mammalian CNS due to molecules such as Nogo $[55,56]$. By contrast, in the fish CNS there is neither Nogo-mediated inhibition [56] nor growth impairment by other glial-cell-associated molecules. Other factors underlying the success or failure of axon regeneration are neuronintrinsic properties including the ability of neurons to reactivate the axon growth program. Fish CNS neurons regenerate axons along with, and supposedly because of, the upregulation of growthassociated proteins $[28,57]$ - including reggie. This ability is poorly developed in mammals. According to recent results [28], reggie/ flotillin upregulation in axotomized neurons is necessary for axon growth - not only in fish but also in mammals (Figure 4). were found first in fish [1], later discovered in mammals $[2,28]$ and, in the latter, in exactly the $3 \%$ of RGCs that, as demonstrated by Alberto Aguayo [45], can regenerate their axon in a growth-permissive environment.

Reggie/flotillin proteins were isolated in coimmunoprecipitation assays with a Thy- 1 antibody, and it was shown that additional, but always specific GPI-linked proteins, namely Thy-1, F3 and PrP, preferentially co-cluster and co-precipitate with reggie/flotillin [31,32]. Furthermore, in neurons just as in $\mathrm{T}$ cells, reggie/flotillin proteins, and with them PrP, Thy- 1 and F3, are associated with the Src tyrosine kinases Src, Fyn and Lck, a finding consistent with the binding of Fyn to reggie/flotillin in fibroblasts [16,41]. Furthermore, reggie/flotillin proteins colocalize with clusters of adaptor and further signaling proteins [30]. Thus, reggies/flotillins seem to represent signaling platforms that are used by proteins with an affinity for the specific lipid composition of the reggie/flotillin microdomains.

Whether the reggie/flotillin proteins are directly and functionally involved in axon regeneration has been assessed in the zebrafish optic nerve. Reggie/flotillin downregulation provoked a clear reduction (up to $70 \%$ ) of the number of regenerating axons, showing that reggies/flotillins are needed for axon regeneration [28]. To correlate this in vivo evidence with the behavior of individual neurons and their attempts to send out axons, reggie expression levels were downregulated in mammalian hippocampal neurons, resulting in striking effects: the neurons failed to differentiate. They either did not form axons or they failed to elongate them. Instead, axons carried abnormal and often immobile club-shaped endings where a growth cone should have been and did not grow [28,47], effects that were partially rescued in the relevant controls. Other neurons had shorter or no processes (Figure 4), were
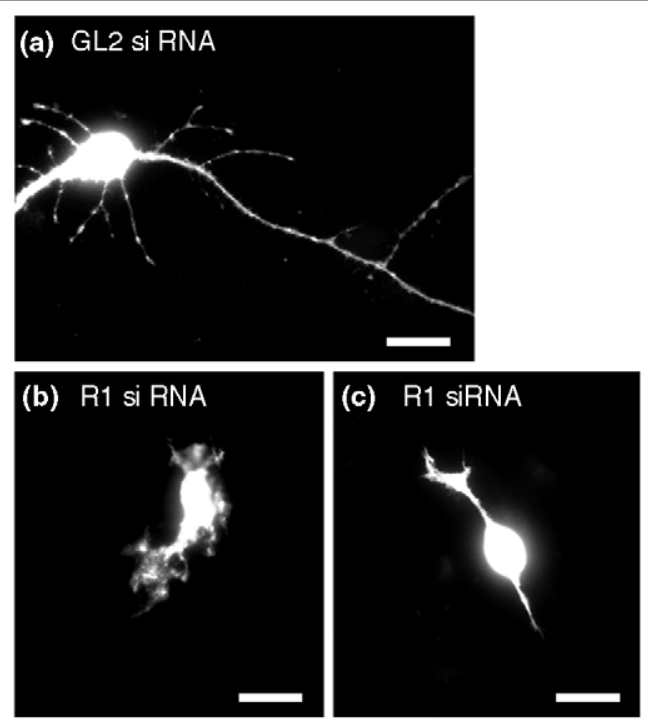

$\overline{\text { TRENDS in Cell Biology }}$

Figure 4. Reggie/flotillin proteins are needed for axon growth. (a) A control siRNAtransfected hippocampal neuron extends a long axon (to the right) and numerous dendrites. (b,c) Hippocampal neurons fail to extend axons and dendrites after treatment with siRNAs targeting reggie-1. According to the hypothesis discussed herein, reggies cluster in growth cones and tips of growing processes to trigger the recruitment of vesicles for the addition of bulk membrane and membrane proteins to the growing processes - an event that ceases after reggie downregulation (modified with permission from Ref. [28]; (c) The Journal of Neuroscience). Bars, $20 \mu \mathrm{m}$. 
abnormally large and malformed, and exhibited conspicuous bulges instead of axons and dendrites [28], implying that the elongation process was blocked because membrane proteins, or more precisely membrane and proteins from the internal vesicle pool, were not appropriately supplied to the prospectively growing tips. Thus, reggie/ flotillin proteins clearly regulate regeneration and axon growth.
Such phenotypes indicate a disturbance in communication with the cytoskeleton, which was next examined in N2a neuroblastoma cells. Indeed, reggie/flotillin mis- and downregulation led to an out-of-balance activation of the Rho-GTPases Rac, Rho and Cdc42. This caused an imbalance in the activation of the downstream effectors WASP, Arp2/3, cortactin and cofilin, and of focal adhesion kinase (FAK), p38 and Ras [28,47,48]. This is compatible with the

(a)

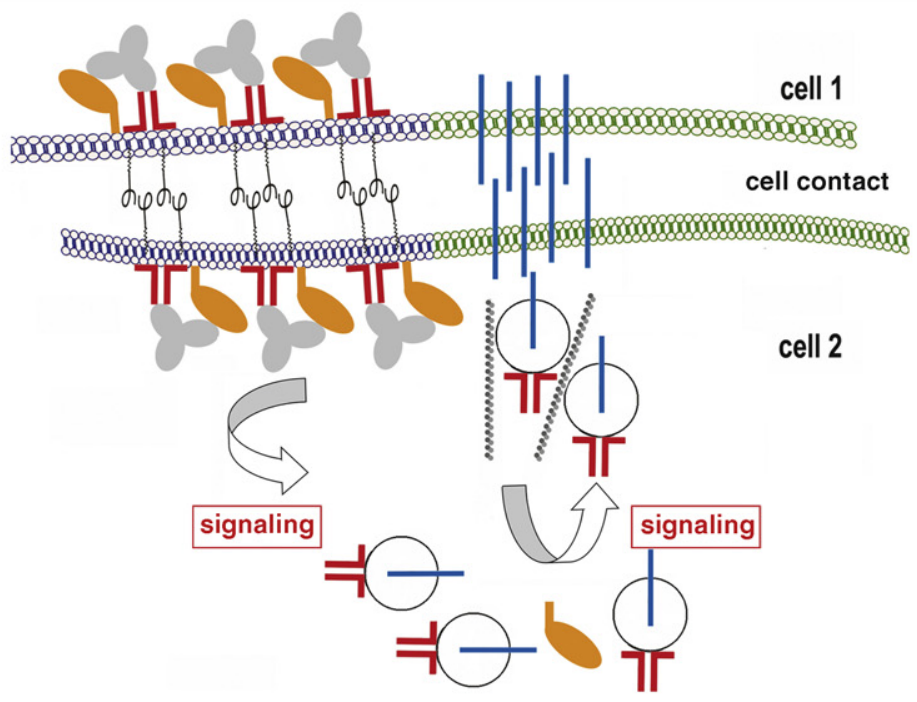

(b)
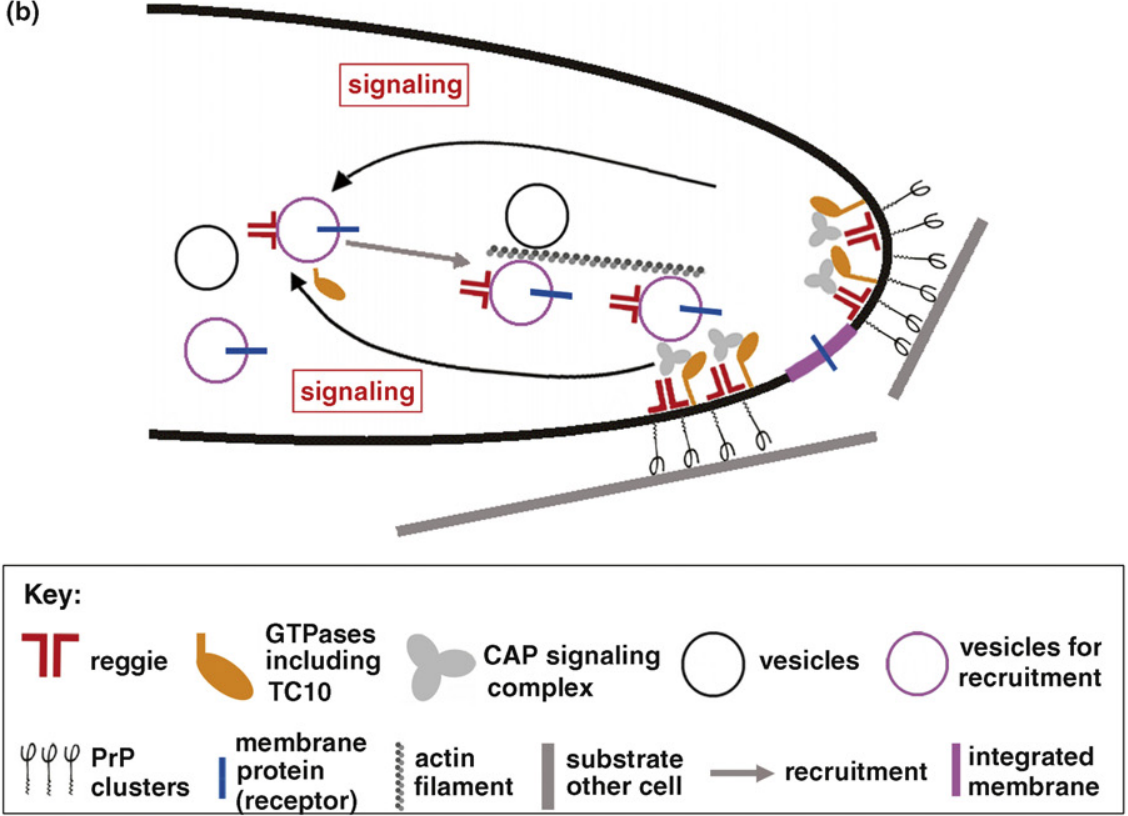

TRENDS in Cell Biology

Figure 5. Reggie/flotillin proteins and the recruitment of membrane proteins. (a) Reggie-1 and reggie-2 (flotillin-2 and -1, respectively) are clustered at cell-cell contact sites and, depending on the cell type, co-cluster with the GPI-anchored proteins Thy-1 and PrP (pig-tailed structures) that are, according to the view detailed herein, involved in the recruitment of cell-adhesion molecules, namely cadherins from vesicles [38,41]. The drawing illustrates these events. PrP activation through trans-interaction at reggiedecorated (red T bars) cell-cell contact sites promotes the activation of small GTPases (orange) such as TC10, through the CAP-associated signaling complex (grey cloverlike complex; see Figure 2 for details). This triggers signal transduction (arrows) to reggie/flotillin-decorated vesicles (circles) associated with the Golgi- or recycling compartments harboring, for instance, cadherin molecules. This involves the GTPases (TC10, Ral, Rac, Rho, Cdc42) and cytoskeletal elements, including actin cables, and results (through the membrane fusion machinery, reggie/flotillin proteins are probably not involved) in membrane insertion of cadherins and homophilic cadherin binding. Cadherin-dependent adhesion might take place in non-raft regions (green) outside reggie/flotillin microdomains (blue), as illustrated in the drawing, whereas a subfraction might insert in or adjacent to reggie/flotillin microdomains, the details of which are still an open question. (b) In the tips of growing processes, recruitment of bulk membrane and membrane proteins can be triggered (black arrows) by interaction of surface proteins (PrP, Thy-1) with the extracellular matrix (ECM) or with other cells (grey lines below and in front of growing tip) functioning as a growth-permissive substrate. This interaction might promote the targeting of membrane and membrane proteins (blue line) from vesicles (violet circle and violet stretch in membrane of the growing tip), such as integrins for cell-substrate interactions and cadherins for cell-cell interactions, to growth cones. 


\section{Box 3. Future questions}

The model proposed here envisages that reggie/flotillin proteins play a role in the targeted delivery of membrane proteins, and in this context future experiments will need to provide conclusive answers to specific questions:

Are reggie/flotillin proteins shuttles? A crucial question concerns exactly how reggie/flotillin microdomains at the plasma membrane communicate with the vesicle pool. Are signals generated by reggie/ flotillin transmitted to vesicles, or are reggie/flotillin shuttles - as suggested by their rapid movement between plasma membrane and vesicle pool [17]? Recordings of reggie/flotillin vesicles might reveal whether they possess a directional preference, for instance during contact formation and axon growth, and whether reggie/ flotillin-dependent trafficking is susceptible to the functional impairment of specific GTPases and Src tyrosine kinases.

Cargo selection, trafficking and targeted delivery. The mechanism by which reggies/flotillins 'select' cargo for the targeted delivery at specific sites (cell contacts, growth cone, T cell cap) is unclear. Whereas reggie/flotillin proteins are absent from the early endosome and from transferrin receptor (TfR)-positive and non-regulated recycling endosomes [17], they are present at the Rab11-positive regulated recycling compartment [58] and on peripheral vesicles loaded with cargo. The introduction of reggie/flotillin mutants [17] that fail to deliver or associate with cargo might lead to an increased understanding of cargo selection and trafficking.

Landmarks? The new view provided here considers that specific GPI-anchored proteins (e.g. PrP), when activated and co-clustered with reggie/flotillin, are landmarks for the targeted delivery of cargo $[33,39]$. If so, then the mechanisms underlying the activation of other GPI-anchored proteins involved in the recruitment of cargo in association with reggie/flotillin must be identified and their communication with vesicle trafficking ascertained.

view that the reggie proteins can affect and modify actin dynamics $[27,30]$ in connection with the recruitment of membrane and proteins (or simply membrane building blocks) from the constitutive secretory pathway and recycling compartment to the growing tips.

\section{A hypothesis explaining reggie/flotillin functions}

Taken together, it seems that reggie/flotillin proteins are crucial for the recruitment of membrane and specific membrane proteins (or membrane building blocks) to specific regions or domains of the cell (Figure 5) - this could well be of great relevance for neurons with elongating and regenerating axons, where reggie/flotillin proteins are enriched at growing tips (Figures 3,5b). Membrane proteins are recruited from vesicular pools (recycling Rab11 compartment, transport vesicles, Golgi-associated vesicles) and moved along cytoskeletal elements, including actin cables [27], a process that involves Rho- and Ras-family small GTPases. The extracellular sensor that triggers reggie/ flotillin-associated signal transduction and membrane protein recruitment, and that also provides specificity, seems to arise from surface molecules with specific affinities for the reggie/flotillin microdomain environment such as GPI-anchored proteins and certain receptor types, including the insulin receptor in adipocytes. The membrane proteins are activated by ligands, binding partners (PrP-PrP trans-interaction), or crosslinking antibodies mimicking ligands, and interact with signaling molecules sharing the preference for reggie/flotillin microdomains, namely Src tyrosine kinases, small GTPases, Cbl and interacting adaptor complexes. This imposes functions on reggie/flotillin proteins in the targeted delivery of mem- brane building blocks with receptors for the growth of cell processes, adhesion, navigation, contact formation and signaling and in sculpturing membrane domains - all clearly important for axon regeneration as well as for growth. A role for the reggie/flotillin proteins in the recruitment of specific vesicles would also account for the dependence of some morphogens ( $\mathrm{wg}, \mathrm{hh}$ ) on reggie in Drosophila.

Furthermore, certain membrane proteins (transporters, receptors, ion channels and adhesion molecules) from vesicles and stores can transiently reside in rafts [23,49] in addition to their normal localization in non-raft membrane. Raft association might result from recruitment through the reggie/flotillin-dependent signaling pathway, implying that reggie/flotillin proteins might act as molecular filters.

The hypothesis outlined above needs further experimental support in many aspects; some of these are discussed in Box 3. It is possible that this role of the reggie/ flotillin proteins - the mediation of the recruitment of proteins/membrane from intracellular pools - requires their existence in all cells, in vertebrate and invertebrate organisms, and makes them key players in axon growth. Upregulation of reggie/flotillin expression is a prerequisite for axon growth/regeneration not only in fish but also in the mammalian central nervous system [2,28]. In fish, all retina ganglion cells express reggie/flotillin at high levels and regenerate their axons. In the mammalian retina, only $3 \%$ of the neurons regenerate their axons and express reggie/flotillin (in contrast to the $97 \%$ that do not and fail to regrow). Because reggie/flotillin proteins are crucial for axon growth (Box 2), perhaps the future will see the development of exciting new strategies to upregulate the expression of reggie/flotillin after neural injury in mammals [50], thus serving as additional therapies after spinal cord injury to encourage neurons to regenerate their axons.

\section{Acknowledgements}

I gratefully acknowledge grants from the Deutsche Forschungsgemeinschaft (DFG) and Fonds der Chemischen Industrie (FCI). I thank in particular Helmut Plattner for the fruitful collaboration, exciting discussions about reggies and helpful critique concerning the manuscript. Thanks also to Ed Malaga-Trillo for comments on the manuscript, Ulrike Binkle for help with the figures and all coworkers, past and present, who devoted time, energy and skills to our reggie research.

\section{References}

1 Schulte, T. et al. (1997) Reggie-1 and reggie-2, two cell surface proteins expressed by retinal ganglion cells during axon regeneration. Development 124, 577-587

2 Lang, D.M. et al. (1998) Identification of reggie-1 and reggie-2 as plasma membrane -associated proteins which co-cluster with activated GPI-anchored cell adhesion molecules in non-caveolar microdomains in neurons. J. Neurobiol. 37, 502-523

3 Rivera-Milla, E. et al. (2006) Ancient origin of reggie (flotillin), reggielike and other lipid raft proteins: convergent evolution of the SPFH domain. Cell. Mol. Life Sci. 63, 343-357

4 Langhorst, M. et al. (2005) Scaffolding microdomains and beyond - the function of reggie/flotillin proteins. Cell. Mol. Life Sci. 62, 2228-2240

5 Hinderhofer, M. et al. (2009) Evolution of prokaryotic SPFH proteins. BMC Evol. Biol. 9, 10

6 Borner, G.H. et al. (2004) Analysis of detergent-resistant membranes in Arabidopsis. Evidence for plasma membrane lipid rafts. Plant Physiol. 137, 104-116 
7 Santamaría, A. et al. (2005) PTOV1 enables the nuclear translocation and mitogenic activity of Flotillin-1, a major protein of lipid rafts. Mol. Cell. Biol. 25, 1900-1911

8 Hazarika, P. et al. (2004) Up-regulation of Flotillin-2 is associated with melanoma progression and modulates expression of the thrombin receptor protease activated receptor. Cancer Res. 64, 7361-7369

9 Katanaev, V. et al. (2008) Reggie-1/flotillin-2 promotes secretion of the long-range signaling forms of wingless and hedgehog in Drosophila. EMBO J. 27, 509-521

10 Babuke, T. and Tikkanen, R. (2007) Dissecting the molecular function of reggie/flotillin proteins. Eur. J. Cell Biol. 86, 525-532

11 Bickel, P.E. et al. (1997) Flotillin and epidermal surface antigen define a new family of caveolae-associated integral membrane proteins. $J$. Biol. Chem. 272, 13793-13802

12 Kirkham, M. et al. (2008) Evolutionary analysis and molecular dissection of caveola biogenesis. J. Cell Sci. 121, 2075-2086

13 Solis, G.P. et al. (2007) Rules for reggie oligomerization and stabilization. Biochem. J. 403, 313-322

14 Glebov, O. et al. (2006) Flotillin-1 defines a clathrin-independent endocytic pathway in mammalian cells. Nat. Cell Biol. 8, 46-54

15 Neumann-Giesen, C. et al. (2007) Role of EGF-induced tyrosine phosphorylation of reggie-1/flotillin-2 in cell spreading and signaling to the actin cytoskeleton. J. Cell Sci. 120, 395-406

16 Riento, K. et al. (2009) Endocytosis of flotillin-1 and flotillin-1 is regulated by Fyn kinase. J. Cell Sci. 122, 912-918

17 Langhorst, M. et al. (2008) Trafficking and association with PrPc of the microdomain proteins Reggie-1 and Reggie-2 (Flotillins). Eur. J. Cell Biol. 87, 211-226

18 Schneider, A. et al. (2008) Flotillin-dependent clustering of the amyloid precursor protein regulates its endocytosis and amyloidogenic processing in neurons. J. Neurosci. 28, 2874-2882

19 Hooper, N.M. et al. (2008) Mechanism of the metal-mediated endocytosis of the prion protein. Biochem. Soc. Trans. 36, 1272-1276

20 Morrow, I.C. and Parton, R.G. (2005) Flotillins and the PHB domain protein family: rafts, worms and anaesthetics. Traffic $6,725-740$

21 Lundmark, R. et al. (2008) The GTPase-activating protein GRAF1 regulates the CLIC/GEEC endocytic pathway. Curr. Biol. 18, 1802-1808

22 Kawase, K. et al. (2006) GTP hydrolysis by the Rho family GTPase TC10 promotes exocytic vesicle fusion. Dev. Cell 11, 411-421

23 Chang, L. et al. (2007) TC10alpha is required for insulin-stimulated glucose uptake in adipocytes. Endocrinology 148, 27-33

24 Chen, X.W. et al. (2006) RalA-exocyst-dependent recycling endosome trafficking is required for the completion of cytokinesis. J. Biol. Chem. 281, 38609-38616

25 Feig, L.A. (2003) RalGTPases: approaching their 15 minutes of fame. Trends Cell Biol. 13, 419-425

26 Lalli, G. and Hall, A. (2005) Ral GTPases regulate neurite branching through GAP-43 and the exocyst complex. J. Cell Biol. 171, 657-869

27 Langhorst, M.F. et al. (2007) Linking membrane microdomains to the cytoskeleton: regulation of the lateral mobility of reggie-1/flotillin-2 by interaction with actin. FEBS Lett. 581, 4697-4703

28 Munderloh, C. et al. (2009) Reggies/flotillins regulate retinal axon regeneration in the zebrafish optic nerve and signal transduction in N2a cells. J. Neurosci. 29, 6607-6615

29 Inoue, M. et al. (2006) Compartmentalization of the exocyst complex in lipid rafts controls Glut4 vesicle tethering. Mol. Biol. Cell 17, 2303 2311

30 Kioka, N. et al. (2002) Vinexin, CAP/ponsin, ArgBP2: a novel adaptor protein family regulating cytoskeletal organization and signal transduction. Cell Struct. Funct. 27, 1-7

31 Dermine, J.F. et al. (2001) Flotillin-1-enriched lipid raft domains accumulate on maturing thagosomes. J. Biol. Chem. 276, 18507-18512

32 Stuermer, C.A. et al. (2001) Glycosylphosphatidyl inositol-anchored proteins and fyn kinase assemble in non-caveolar plasma membrane microdomains defined by reggie-1 and reggie-2. Mol. Biol. Cell 12, 3031-3045

33 Stuermer, C.A. et al. (2004) $\mathrm{PrP}^{\mathrm{c}}$ capping in $\mathrm{T}$ cells promotes its association with the lipid raft proteins reggie-1 and reggie-2 and leads to signal transduction. FASEB J. 18, 1731-1733
34 Rajendran, L. et al. (2003) Asymmetric localization of flotillins/reggies in pre-assembled platforms confers inherent polarity to hematopoetic cells. Proc. Natl. Acad. Sci. U. S. A. 100, 8241-8246

35 Langhorst, M. et al. (2006) Preformed reggie/flotillin caps: stable priming platforms for macrodomain assembly in T cells. FASEB $J$. 20, 711-713

36 Simons, K. and Ehehalt, R. (2002) Cholesterol, lipid rafts, and disease. J. Clin. Invest. 110, 597-603

37 Das, V. et al. (2004) Activation-induced polarized recycling targets T cell antigen receptors to the immunological synapse: involvement of the SNARE Complexes. Immunity 20, 577-588

38 Villalba, M. et al. (2001) VAV1/Rac-dependent actin cytoskeleton reorganisation is required for lipid raft clustering in T cells. J. Cell Biol. 155, 331-338

39 Malaga-Trillo, E. et al. (2009) Regulation of embryonic cell adhesion by the prion protein. PLOS Biol. 7, e1000055

40 Simons, K. and Vaz, L.C. (2004) Model systems, lipid rafts, and cell membranes. Annu. Rev. Biophys. Biomol. Struct. 33, 269-295

$41 \mathrm{Liu}$ et al. (2005) The stomatin/prohibitin/flotillin/HflK/C domain of flotillin-1 contains distinct sequences that direct plasma membrane localization and protein interactions in 3T3-L1 adipocytes. $J$ Biol. Chem. 280, 16125-16134

42 Chiesa, R. and Harris, D.A. (2009) Fishing for prion protein function. PLoS Biol. 7, e1000075

43 Gaze, R.M. (1970) Formation of Nerve Cell Connections, Academic Press

44 Taylor, A.M. et al. (2009) Axonal mRNA in uninjured and regenerating cortical mammalian axons. J. Neurosci. 29, 4697-4718

45 Richardson, P.M. et al. (1980) Axons from CNS neurons regenerate into PNS grafts. Nature 284, 264-265

46 Stuermer, C.A.O. and Plattner, H. (2005) The 'lipid raft'/microdomain proteins reggie-1 and reggie-2 (flotillins) form scaffolds for protein interactions and signaling. Biochem. Soc. Symp. 72, 109-118

47 Langhorst et al. (2008) Reggies/flotillins regulate Rho GTPase signalling during axon outgrowth and axon regeneration. Eur. J. Cell Biol. 87, 921-931

48 Schrock, Y. et al. (2009) Regulation of cell spreading, focal adhesion formation and filopodia extension by the cellular prion protein. FEBS Lett. 583, 389-393

49 Rossy, J. et al. (2009) Flotillins interact with PSGL-1 in neutrophils and, upon stimulation, rapidly organize into membrane domains subsequently accumulating in the uropod. Plos One 4, 5403

50 Sasaki, Y. et al. (2008) Identification of flotillin-2, a major protein on lipid rafts, as a novel target of p53 family members. Mol. Cancer Res. 6 , 395-406

51 Morrow, I.C. et al. (2002) Flotillin-1/reggie-2 traffics to surface raft domains via a novel golgi-independent pathway. Identification of a novel membrane targeting domain and a role for palmitoylation. $J$. Biol. Chem. 277, 48834-48841

52 Miyamoto, K. et al. (2004) Solution structure of the band 7 domain of the mouse flotillin 2 protein. RSCB Protein Data Bank, http:// www.pdb.org/pdb/explore.do?structureId=1WIN

53 Browman, D.T. et al. (2007) The SPFH domain-containing proteins: more than lipid raft markers. Trends Cell Biol. 17, 394-402

54 Reuter, A. et al. (2004) $\mathrm{PrP}^{\mathrm{c}}$ and Reggies/Flotillins are contained in and released via lipid-rich vesicles in Jurkat T cells. Cell. Mol. Life Sci. 61, 2092-2099

55 Maier, I.C. and Schwab, M.E. (2006) Sprouting, regeneration and circuit formation in the injured spinal cord: factors and activity. Phil. Trans. R. Soc. B Biol. Sci. 361, 1611-1634

56 Diekmann et al. (2005) Evidence for the absence of the neurite growth inhibitory protein Nogo-A in fish. J. Mol. Evol. 22, 16351648

57 Benowitz, L.I. and Yin, Y. (2007) Combinatorial treatments for promoting axon regeneration in the CNS: strategies for overcoming inhibitory signals and activating neurons' intrinsic growth state. Dev. Neurobiol. 67, 1148-1165

58 Grant, B.D. and Donaldson, J.G. (2009) Pathways and mechanisms of endocytic recycling. Nat. Rev. Mol. Cell Biol. 10, 597-608 